\title{
1980 Microprocessor Users' Group meeting report
}

\author{
M. PAVEL \\ New York University, New York, New York 10003
}

The users' group meeting included a presentation of the results of the computer users survey, a demonstration of a microprocessor-based experiment, and a general discussion.

The survey, whose results appear in Section XIII of this issue, was in part carried out to determine the needs of the microprocessor users. As expected, the types of equipment, systems, and software vary a great deal among the users who responded to the questionnaire. This fact accounts for the difficulty the group has in satisfying a large proportion of users at any one meeting.

Joseph Psotka presented a demonstration based on the Radio Shack TRS-80 system in which he focused on the ability of human subjects to produce random sequences. Since both stimulus presentation and collection of responses were mediated by a terminal, there was no need for special interface hardware. Thus this demonstration, which stimulated considerable interest, was an excellent example of an efficient application of low-cost microprocessor technology.

Most of the problems brought up during the group's general discussion were related to the ways in which the
Microprocessor Users' Group (MUG) could help solve everyday problems. Most of those who attended this meeting seemed to agree that the most effective function of the MUG is to maintain a data base of equipment and software used by psychologists. Such a data base should be kept up-to-date and be easily accessible to a wide population. Despite the concensus on this issue, there were no volunteers to undertake the project.

Compiling such a data base begins with the development, distribution, and collection of a sufficiently detailed questionnaire. Even though we have no specific design for the eventual user-wide access to the data base as yet, we are in the process of collecting the relevant information. I hope that we will get the required cooperation of all users in promptly retuming completed questionnaires.

Another issue briefly discussed concerned the question of combining the microprocessor and the DEC users' groups. While the results of the survey indicated a slight preference for this merger, some of the users present at the meeting felt that such a move is premature. At this time, no final decision has been made. 